\title{
In-Plane fracture of laminated fiber reinforced composites with varying fracture resistance: experimental observations and numerical crack propagation simulations
}

\author{
Siva Shankar Rudraraju* Amit Salvi† Krishna Garikipati ${ }^{\ddagger}$ and Anthony M. Waas ${ }^{\S}$ \\ College of Engineering, University of Michigan, Ann Arbor, MI 48109, USA
}

\begin{abstract}
A series of experimental results on the in-plane fracture of a laminated composite panel is analyzed using the variational multi-scale cohesive method (VMCM). The VMCM results demonstrate the influence of specimen geometry and load distribution on the propagation of large scale bridging cracks in laminated fiber reinforced composites. Experimentally observed variation in fracture resistance is substantiated numerically by comparing the experimental and VMCM load-displacement responses of geometrically scaled single edgenotch three point bend (SETB) specimens. The results elucidate the size dependence of the traction-separation relationship for this class of materials even in moderately large specimens, contrary to the conventional understanding of it being a material property. The existence of a "free bridging zone" (different from the conventional "full bridging zone") is recognized, and its influence on the evolving fracture resistance is discussed. The numerical simulations and ensuing bridging zone evolution analysis demonstrates the versatility of VMCM in objectively simulating progressive crack propagation, compared against conventional numerical schemes like traditional cohesive zone modeling, which require a priori knowledge of the crack path.
\end{abstract}

\section{Introduction}

Laminated fiber reinforced composites are finding increased use as structural materials in a variety of aerospace and other industrial applications. An appealing property of these composites is their high specific strength (strength per unit weight). Even though a strong case can be made for using composite structural parts in several areas of a load bearing structure, the requirement to demonstrate structural integrity and damage tolerance (SIDT) necessitates a fundamental understanding of the mechanical response, damage tolerance and damage growth of a load bearing composite structure. While several investigations have addressed damage growth in the form of delamination crack growth, much less is known or understood about damage propagation when a crack, or damage in the form of a crack that has severed all laminae of a laminate, is present in a composite structure. The influence of such wide area damage on the load bearing ability of a homogeneous and isotropic material has received considerable attention in the past; however, a similar effort at understanding issues in a non-homogeneous and macroscopically orthotropic structure is still a problem that requires resolution. Because of the different length scales associated with the microstructure of a composite material and the resulting composite structure, a multitude of failure mechanisms can be simultaneously operative, leading to a very complex damage progression in a composite structure. A sharp, through the thickness crack can be present in these composites initially, but, as soon as local damage (this can be in the form of matrix micro-cracking) accumulates, crack blunting and distributed damage occurs across the highly stressed areas around the initial crack tip. As this initial crack starts to grow, a damaged zone of material (bridging zone) evolves in the wake of the instantaneous crack tip. Thus,

\footnotetext{
${ }^{*}$ Graduate Student Research Assistant, Department of Aerospace Engineering.

${ }_{\dagger}^{\dagger}$ Research Fellow, Department of Aerospace Engineering.

$\ddagger_{\ddagger}^{\ddagger}$ Associate Professor, Department of Mechanical Engineering.

$\S$ Professor, Department of Aerospace Engineering and Department of Mechanical Engineering. Associate Fellow, AIAA.
} 
unlike in monolithic materials (such as metals), there is no clean "crack" that can be identified. Instead, a diffused zone of damage is seen to advance. This distributed damage results in additional resistance for advancing damage growth, largely contributed by fiber bridging in the crack wake. This enhanced fracture resistance is desirable and is a major contributor to the increased toughness of these laminated composites. ${ }^{1-4}$

Analytical models based on linear elastic fracture mechanics (LEFM) have been developed and implemented within finite element codes to study a variety of fracture problems. Essential to the success of LEFM approaches is the requirement of a small process zone ahead of the crack tip. ${ }^{34}$ With negligible material non-linearity at the crack tip (small process zone size), LEFM based approaches have proven to be effective in predicting crack initiation and subsequent growth. ${ }^{34-38}$ However, in heterogeneous materials like laminated fiber composites, the process zone size may be larger than any characteristic problem length scale, and thereby, the basic tenets of LEFM cease to hold. ${ }^{41}$ Several mechanisms may contribute to this situation. Micro-cracking, fiber bridging, coalescence of voids and other microstructural mechanisms can give rise to a process zone that is considerably larger than that permitted for the application of LEFM models. A new length scale, $l^{*}$, emerges that is related to a characteristic elastic modulus $E$, fracture toughness $\Gamma$ and cohesive strength, $\sigma_{c}$, defined as, $l^{* 2}=\frac{E \Gamma}{\sigma_{c}^{2}}$. If $l^{*}$ is larger than any characteristic length scale in the problem, then, cohesive zone models (CZM) become an indispensable tool for analysis. ${ }^{28-33}$

In order to implement a CZM in its simplest form, two fracture parameters are required: a fracture toughness (or energy) and a cohesive strength. Both parameters are experimentally determined by coupon level tests, and are subsequently used as material properties for prediction of crack growth in other structural configurations. In CZM, an existing crack starts to grow when the crack tip stresses reach the cohesive strength, and evolves according to the energy available in the deformed system to create additional cracked surface. Based on these two parameters, a cohesive traction-separation law is assumed. This micro-mechanical law, relates the evolution of crack face tractions with the crack face opening displacement, and numerically manifests the resistance offered to crack advancement within the cohesive zone. This two parameter dependent evolution, unlike the solely fracture toughness based LEFM schemes, better represents the physics of crack growth in materials with significant process zones, and it has been widely used in many numerical implementations, like the discrete cohesive zone method (DCZM). ${ }^{39,41}$ However, all implementations of CZM methodology require a priori knowledge of the "intended" crack path. This prevents CZM from being applied to a wide range of problems involving arbitrary crack path evolution. Conventional finite element based implementations of $\mathrm{CZM}^{20}$ constrain the crack path evolution to element interfaces, as standard finite elements lack the ability to represent cracks within the element domain. These restrictions introduce significant mesh dependency into the problems; an aspect that defeats the requirements of a predictive analysis tool.

In order to circumvent these numerical restrictions on unrestricted and objective simulation of crack propagation in materials, a micro-mechanics based, mesh independent numerical technique for simulating crack propagation is essential. Standard finite elements fail to accomplish this task as they lack the ability to capture the discontinuous displacement modes involved in crack propagation problems. However, in recent years finite elements with discontinuities (enhanced finite elements) have gained increasing interest in modeling material failure, due to their ability to capture the specific kinematics of a displacement discontinuity(like cracks) through additional discontinuous deformation modes. In discontinuous displacement enhanced finite elements the crack path is present inside the elements, unlike cohesive zone methods which are restricted to crack propagation along element interfaces. The ability of the enhanced finite element to encompass a crack path, leads to objective simulation of crack propagation without any mesh bias. Depending on the support of the enriching discontinuous displacement modes, the enhanced finite elements are popularly known as enhanced strain finite elements (EFEM- elemental enrichment) $)^{9,12-17}$ and extended finite elements (XFEM - nodal enrichment). ${ }^{10,11,18,19}$ Interested readers are referred to Oliver et al., ${ }^{21}$ for detailed discussion and comparison of these methods. Though these enhanced methods provide a general numerical framework for simulation of crack evolution, the actual micromechanics implementation which incorporates the physics of crack formation is wide open. In this context, we present the Variation Multiscale Cohesive Method (VMCM), which is an enhanced finite element method containing elemental displacement field enrichment, naturally arising out of the variational multiscale formulation presented in Garikipati et al. ${ }^{8,12}$ seamlessly embedding the cohesive nature of crack path evolution. In this paper, the VMCM method is briefly presented and it is applied to simulate crack propagation in a laminated fiber reinforced composite 
panel. A more detailed presentation of VMCM is available in other related studies by the authors, ${ }^{8,22}$ where the method is referred to as the variational multiscale method (VMM).

The paper is organized as follows: In section II, the application of VMCM to study crack propagation is motivated through a discussion of experimental results in scaled SETB specimens, loaded to failure. Section III presents the details of VMCM implementation using the finite element method. Simulations of SETB tests with varying specimen size are provided in section IV. Comparison of experimental results, against the predictions of VMCM are discussed in section $\mathrm{V}$, while concluding remarks are presented in section VI.

\section{Motivation}

The primary motivation for the current work is the experimentally observed scaling in the in-plane fracture resistance $^{\mathrm{a}}$, with increase in specimen size of single edge notch three point bend (SETB) specimen, subjected to primarily Mode-I crack tip conditions. The material used in all the experiments herein is a $[-45 / 0 /+45 / 90]_{6 s}$ laminated fiber reinforced composite cut from large panels.

The SETB configuration used in this study is shown in Figure 1. Five specimen sizes with geometrically scaled planar geometry and fixed thickness are considered. Multiple specimens of each size were tested to significantly capture the failure response envelope. The load - load point displacement $(P \Delta)$ responses of these specimens are shown in Figure 4. The in-plane fracture resistance of each specimen is calculated by normalizing the total area under the $P \Delta$ curve by the area (thickness times total crack length) of the crack surface formed. But the multiple tests for each SETB size show significantly varying $P \Delta$ response. Hence, an averaged fracture resistance, $\mathscr{R}_{a v g}$, of each specimen is calculated by averaging the individual in-plane fracture resistance values obtained from each of the $P \Delta$ curves of each specimen ${ }^{\mathrm{b}}$. As will be demonstrated and discussed in Section IV, $\mathscr{R}_{\text {avg }}$ captures the correct failure response, and it is much easier to calculate.

Table 1 summarizes the observed scaling in the $P \Delta$ response, and hence in the value of $\mathscr{R}_{\text {avg }} . P^{*}, \Delta^{*}$ and $\mathscr{R}_{\text {avg }}^{*}$ are fixed reference values. $\mathscr{R}_{\text {avg }}^{*}$ is the value of fracture resistance obtained in similar tests on standard Compact Tension (CT) specimens (Figure 3(a)). The scaling observed in $\mathscr{R}_{\text {avg }}$ is very significant, because in all the specimen sizes considered, it has been experimentally observed that there is formation of a Full Bridging Zone $(\mathrm{FBZ})^{\mathrm{c}}$ (Figure 2), and the length of FBZ scales up with specimen geometry. The formation of a FBZ in all specimen sizes considered and the observed scaling in its length are contrary to the usual expectation, wherein, there is no FBZ formation prior to complete failure in smaller specimens, and when FBZ formation does occur in large enough specimens, the FBZ length is a material property. This assumption of a constant length FBZ formation can lead to incorrect predictions of strength and reliability, because in some classes of materials, like the one being considered here, a FBZ formation can be misinterpreted as the formation of a converged process zone, whereas, in reality, larger size specimens might still lead to longer process zones, and hence greater FBZ length. In order to substantiate and understand the mechanics behind such scaling behavior and FBZ formation, VMCM based simulations were conducted. Before proceeding to the numerical simulations, the VMCM framework is briefly presented in the following section.

a Traditionally, fracture resistance is defined in terms of the energy released per unit area of crack surface formation. But, unlike in most monolithic materials, there is no clean "crack" that can be identified in a laminated fiber composite panel, where a crack like feature propagates severing all laminae of the laminate. Then, by unit area of crack surface, we mean unit area of completely failed (both matrix and fiber failure) surface along the "crack" like diffused zone of damage. The usage of the term "fracture toughness" is resisted, to avoid confusion with the traditional LEFM approach, where it is often regarded as a material property.

${ }^{b}$ One may suggest the R-curves approach of LEFM as a better alternative. However the failure response of this material is very stochastic, and lacks post-peak $P \Delta$ response repeatability, which is important for obtaining consistent R-curves. Averaging R-curves across specimens of each size is possible, but it is of little advantage over $\mathscr{R}_{\text {avg }}$. Further, the total energy released may include dissipation due to other mechanisms, like crack tip plasticity, but for this class of materials the crack formation energy is the predominant component of the total energy dissipated.

${ }^{\mathrm{C}}$ Full Bridging Zone (FBZ) is assumed to have formed when fibers at the location of the initial crack tip have failed/completely pulled out, leading to zero tractions across the crack faces at the initial crack tip. ${ }^{4}$ FBZ formation is also referred to as the formation of a "Stable Process Zone". When the fibers at the initial crack tip have not yet failed, the bridging zone is still evolving, and herein we refer to this as the partial bridging zone. 


\section{Mathematical Formulation}

The standard weak form of the balance of linear momentum is given by,

$$
\int_{\Omega} \nabla^{s} \boldsymbol{w}: \boldsymbol{\sigma} \mathrm{dV}=\int_{\Omega} \boldsymbol{w} \cdot \boldsymbol{b} \mathrm{dV}+\int_{\partial \Omega_{t}} \boldsymbol{w} \cdot \boldsymbol{T} \mathrm{dS} .
$$

where $\boldsymbol{\sigma}$ is the stress, $\boldsymbol{w}$ is the admissible displacement variation, $\nabla^{s} \boldsymbol{w}$ is the symmetric gradient of the variation, $\boldsymbol{T}$ is the external traction and $\boldsymbol{b}$ is the body force. $\Omega$ represents the domain of the problem (Figure 6 ).

In the standard finite element formulation of continuum mechanics, the displacements are at least $C^{0}$ continuous. But in a wide class of problems (shear banding, fiber kink banding, transverse crack formation, delamination initiation are some examples), the displacement field can be discontinuous. In such cases, the displacement field can be decomposed into continuous coarse scale and discontinuous fine scale components (Figure 5). This decomposition is written as,

$$
\boldsymbol{u}=\underbrace{\overline{\boldsymbol{u}}}_{\text {coarse scale }}+\underbrace{\boldsymbol{u}^{\prime}}_{\text {fine scale }}
$$

The corresponding scale separation in the displacement variation is given by,

$$
\boldsymbol{w}=\underbrace{\overline{\boldsymbol{w}}}_{\text {coarse scale }}+\underbrace{\boldsymbol{w}^{\prime}}_{\text {fine scale }}
$$

Substituting the above decomposition into (1), and using standard arguments, the weak form can be split into two separate weak forms. One, involving the coarse scale variation, $\overline{\boldsymbol{w}}$, and the other, involving only the fine scale variation, $\boldsymbol{w}^{\prime}$.

$$
\begin{gathered}
\int_{\Omega} \nabla^{s} \overline{\boldsymbol{w}}: \boldsymbol{\sigma} \mathrm{dV}=\int_{\Omega} \overline{\boldsymbol{w}} \cdot \boldsymbol{b} \mathrm{dV}+\int_{\partial \Omega_{t}} \overline{\boldsymbol{w}} \cdot \boldsymbol{T} \mathrm{dS} . \\
\int_{\Omega^{\prime}} \nabla^{s} \boldsymbol{w}^{\prime}: \boldsymbol{\sigma} \mathrm{dV}=\int_{\Omega^{\prime}} \boldsymbol{w}^{\prime} \cdot \boldsymbol{b} \mathrm{dV}+\int_{\partial \Omega_{t}^{\prime}} \boldsymbol{w}^{\prime} \cdot \boldsymbol{T} \mathrm{dS} .
\end{gathered}
$$

This procedure results in the fine scale weak form (5), defined only over $\Omega^{\prime}$ (Figure 6). This result is crucial since it lends itself naturally to the application of desired micromechanical descriptions restricted to the microstructural region, $\Omega^{\prime}$, and not the entire domain $\Omega$. The scale separation in $u$ is contained in $\sigma=\mathcal{C}:\left(\nabla^{s} \overline{\boldsymbol{u}}+\nabla^{s} \boldsymbol{u}^{\prime}\right)$, where $\mathcal{C}$ is the elastoplastic tangent.

We wish to use an appropriate micromechanical law by which the fine scale solution, $\boldsymbol{u}^{\prime}$, can be expressed in terms of $\overline{\boldsymbol{u}}$ and other fields in the problem. Below, we will show how such a micromechanical law can be embedded into the formulation using the weak form (5). The final step involves elimination of the fine scale displacement, $\boldsymbol{u}^{\prime}$, from the problem by substituting its relation to $\overline{\boldsymbol{u}}$ in the coarse scale weak form (4). Thus, the fine scale solution does not appear explicitly; however, its effect is fully embedded in the resultant modified weak form.

We choose $\Omega^{\prime}$ to contain the crack surface $\Gamma$ on which $\boldsymbol{u}^{\prime}$ is discontinuous. Invoking standard variational arguments, the weak form of the fine scale problem can be reduced to the following statement of traction continuity:

$$
\llbracket \boldsymbol{\sigma} \boldsymbol{n} \rrbracket_{\Gamma}=0
$$

where $\llbracket . \rrbracket$ is the discontinuity in the quantity and $\boldsymbol{n}$ is the normal to the crack surface, $\Gamma$. Writing the traction on $\Gamma$ in terms of components $T_{n}$ and $T_{m}$ along $\boldsymbol{n}$ and $\boldsymbol{m}$ respectively (Figure 6), the traction continuity condition can be expressed as,

$$
T_{n} \boldsymbol{n}+T_{m} \boldsymbol{m}=\left.\boldsymbol{\sigma} \boldsymbol{n}\right|_{\Gamma^{-}}
$$

The traction $\left.\boldsymbol{\sigma} \boldsymbol{n}\right|_{\Gamma^{-}}$, is determined by the macromechanical continuum formulation. The evolution of $T_{n}$ and $T_{m}$ is governed by the assumed micromechanical surface law (Section IV(A)). The displacement 
discontinuity $\llbracket \boldsymbol{u} \rrbracket$ can be expressed in terms of the normal opening, $\llbracket \boldsymbol{u} \rrbracket . n$, and tangential slip, $\llbracket \boldsymbol{u} \rrbracket . \boldsymbol{m}$, across $\Gamma$.

We now consider a general functional form for the micromechanical model,

$$
T_{n}=T_{n_{0}}-\mathcal{H}_{n} \llbracket \boldsymbol{u} \rrbracket . \boldsymbol{n}, \quad T_{m}=T_{m_{0}}-\mathcal{H}_{m} \llbracket \boldsymbol{u} \rrbracket . m
$$

where $T_{n_{0}}>0, T_{m_{0}}>0$ are the traction components at $\llbracket \boldsymbol{u} \rrbracket \cdot \boldsymbol{n}=0, \llbracket \boldsymbol{u} \rrbracket \cdot \boldsymbol{m}=0$, respectively, and $\mathcal{H}_{n}, \mathcal{H}_{m}$ are the corresponding softening parameters. Their physical interpretations are discussed in Section IV(A). Consistency between the micromechanical law and the macromechanical continuum description is enforced by (7) via (8).

Substituting (8) in (7) and dispensing with the explicit indication of $\left.\boldsymbol{\sigma} \boldsymbol{n}\right|_{\Gamma^{-}}$,

$$
\left(T_{n_{0}}-\mathcal{H}_{n}(\llbracket \boldsymbol{u} \rrbracket . \boldsymbol{n})\right) \boldsymbol{n}+\left(T_{m_{0}}-\mathcal{H}_{m}(\llbracket \boldsymbol{u} \rrbracket . \boldsymbol{m})\right) \boldsymbol{m}-\boldsymbol{\sigma} \boldsymbol{n}=0
$$

Expanding (9) up to first order terms, in order to solve for $\boldsymbol{u}^{\prime}$ :

$$
\begin{aligned}
&\left(T_{n_{0}}-\mathcal{H}_{n}(\llbracket \boldsymbol{u} \rrbracket . \boldsymbol{n})\right) \boldsymbol{n}+\left(T_{m_{0}}-\mathcal{H}_{m}(\llbracket \boldsymbol{u} \rrbracket . \boldsymbol{m})\right) \boldsymbol{m}-\boldsymbol{\sigma} \boldsymbol{n} \\
&-\mathcal{H}_{n}(\delta \llbracket \boldsymbol{u} \rrbracket . \boldsymbol{n}) \boldsymbol{n}-\mathcal{H}_{m}(\delta \llbracket \boldsymbol{u} \rrbracket . \boldsymbol{m}) \boldsymbol{m} \\
&-\left(\mathcal{C}:\left(\nabla \delta \overline{\boldsymbol{u}}+\nabla \delta \boldsymbol{u}^{\prime}\right)\right) \boldsymbol{n}=0
\end{aligned}
$$

where the first line in (10) represents a zeroth-order approximation to (9), and the remaining terms are the first order corrections. $\mathcal{C}$ is the elastic tangent modulus. Using $\boldsymbol{u}^{\prime}=\llbracket \boldsymbol{u} \rrbracket C_{\Gamma}$, where $C_{\Gamma}$ is the fine scale interpolation (Figure 7), converts (10) into a linear equation in $\delta \llbracket \boldsymbol{u} \rrbracket$ which can be solved, and then the incremental fine scale field is obtained from $\delta \boldsymbol{u}^{\prime}=\delta \llbracket \boldsymbol{u} \rrbracket \boldsymbol{C}_{\Gamma}$. Formally, it is represented as,

$$
\delta \boldsymbol{u}^{\prime}=F\left[\overline{\boldsymbol{u}}, \boldsymbol{\sigma}, T_{n}, T_{m}, \xi_{n}, \xi_{m}\right]
$$

Noting that $\boldsymbol{\sigma}$ in a general nonlinear problem can itself be expanded up to first order as $\boldsymbol{\sigma}=\boldsymbol{\sigma}+$ $\mathcal{C}:\left(\nabla \delta \overline{\boldsymbol{u}}+\nabla \delta \boldsymbol{u}^{\prime}\right)$, and substituting $\boldsymbol{u}^{\prime}$ in (4), we obtain the coarse field weak form which is independent of the fine scale displacement $\boldsymbol{u}^{\prime}$. On solving for $\delta \overline{\boldsymbol{u}}$, the incremental fine scale field $\delta \boldsymbol{u}^{\prime}$ can be recovered via (11). Iterations are to be performed: $\overline{\boldsymbol{u}}^{(i+1)}=\overline{\boldsymbol{u}}^{(i)}+\delta \overline{\boldsymbol{u}}, \boldsymbol{u}^{(i+1)}=\boldsymbol{u}^{(i)}+\delta \boldsymbol{u}^{\prime}$, until a converged solution is obtained. From (4),(10) and (11), it should be clear that the VMCM method results in an embedding of the micromechanical surface law into the coarse scale weak formulation. Interested readers are referred to Garikipati, ${ }^{8}$ for a more detailed discussion of the numerical framework.

Having briefly presented the numerical formulation, we direct our attention to simulations, and the mechanisms involved in the in-plane fracture of laminated fiber composites.

\section{Numerical Simulations}

\section{A. Micromechanical surface law}

The micromechanics of crack propagation is embedded into the macroscopic formulation of the VMCM by enforcing the following traction evolution laws (9),

$$
\begin{aligned}
T_{n} & =T_{n_{0}}-\mathcal{H}_{n} \llbracket \boldsymbol{u} \rrbracket . \boldsymbol{n} \\
T_{m} & =T_{m_{0}}-\mathcal{H}_{m} \llbracket \boldsymbol{u} \rrbracket . \boldsymbol{m}
\end{aligned}
$$

where $T_{n_{0}}>0$ and $T_{m_{0}}>0$ are the maximum values of $T_{n}$ and $T_{m}$ admissible on $\Gamma$ (Figure 6 ), $\llbracket \boldsymbol{u} \rrbracket . n>0$ and $\llbracket \boldsymbol{u} \rrbracket . \boldsymbol{m}>0$ are the normal jump (Mode-I type crack opening) and tangential slip (Mode-II type crack face slip) across the elemental crack face, $\mathcal{H}_{n}$ and $\mathcal{H}_{m}$ are the softening moduli for the Mode-I and Mode-II crack opening evolution, respectively. 
Due to the stochastic behavior observed in experiments and nonlinearity of response (Section V), it is difficult to obtain precise functional forms for $\mathcal{H}_{n}$ and $\mathcal{H}_{m}$. The accepted methodology in the cohesive zone community ${ }^{25-27}$ is to assume a suitable functional form of the traction evolution response, referred to as the traction separation law, anchored by experimentally determined values of $T_{n_{0}}$, Mode-I $\mathscr{R}_{\text {avg }}$ and $T_{m_{0}}$, Mode-II $\mathscr{R}_{\text {avg. }}$. In the current study, a linear traction separation law is assumed for the evolution of $T_{n}{ }^{\mathrm{d}}$ (Figure 8). There is no theoretical or physical rationale in assuming a linear traction separation law as against any other functional form ${ }^{\mathrm{e}}$. The actual shape of the traction separation law is still a topic of active research $^{24}$ and some methods have been devised to measure the law for a limited class of configurations. ${ }^{43,44}$

The value of $T_{n_{0}}$ is obtained by experiments on double notched tension specimens (Figure 3(b)). This configuration was selected because the stress state across the entire crack face is almost uniform and specimen failure is instantaneous. Thus, the critical load divided by the total crack area gives a fairly accurate estimate of the critical traction across the crack faces. It was observed that this value was independent of the specimen size. The $\mathscr{R}_{\text {avg }}$ values are given in Table (1). With these two inputs, the five SETB specimen sizes given in Figure 1 are simulated using the VMCM.

\section{B. Simulations}

The VMCM methodology is based on the ability of the fine scale displacement field to capture displacement discontinuities, like cracks. Figure 10 shows a typical SETB mesh of triangular VMCM elements in the reference and deformed states. In Figure 10(b), the highly deformed elements in the inset are the ones through which the crack passes. These elements appear to have large strains because the singular strain field is effectively smeared over the element by plotting in the deformed configuration. As the crack propagates, the elements containing the crack deform and the normal crack opening $\llbracket \boldsymbol{u} \rrbracket . n>0$ increases, and thereby ultimately leading to zero normal tractions across the crack faces and hence loss of normal load bearing capacity of the element. The same happens with the evolution of the tangential tractions. After the element loses the ability to bear both normal and tangential tractions, the mesh may be post-processed to remove these failed elements, to present a visually appealing crack propagation.

The criterion selected in this work for validating the VMCM methodology is the comparison of VMCM and experimental $P \Delta$ responses of SETB specimens (Figure 1). This criterion is chosen because the $P \Delta$ response reflects the macroscopic response of the structure to external loads, and often in structural design the peak load is the value of primary interest. Figure 11 shows the $P \Delta$ curves extracted from VMCM simulations of SETB specimens with a constant, $\mathscr{R}_{\text {avg }}=\mathscr{R}_{\text {avg }}^{*}$, input for all sizes considered. As seen in the figure, the load displacement response is significantly captured for smaller specimens (because $\mathscr{R}_{\text {avg }}^{*}$ is close to $m a t h s c r R_{\text {avg }}$ / value for Size-1 and Size-2 specimens), but the peak load is severely under-predicted for larger specimens. This is expected, as the experimental values of $\mathscr{R}_{\text {avg }}$ for large specimens are much higher then the input value $\mathscr{R}_{\text {avg }}^{*}$ (Table 1$)$. Now the simulations are conducted with the experimentally measured $\mathscr{R}_{\text {avg }}$ values given in Table 1, and the results are plotted in Figure 12.

As seen from Figure 12, the VMCM simulations accurately reproduce the macroscopic response of the SETB specimens when appropriate $\mathscr{R}_{\text {avg }}$ values are used as input ${ }^{\mathrm{f}}$. This demonstrates that: (1) The VMCM methodology has the ability to numerically simulate progressive damage propagation, and the mechanics of bridged crack evolution. (2) In spite of a multitude of failure mechanisms operating simultaneously, leading to a very complex evolution of fracture resistance, the averaging of overall fracture resistance leads to a useful, single valued estimate, $\mathscr{R}_{a v g}$, of the fracture resistance appropriate for numerical simulations, at least

\footnotetext{
${ }^{\mathrm{d}}$ In order to avoid additional physical complexities arising from mode mixity conditions only test cases undergoing pure Mode-I evolution are considered in the current study, and hence there is no significant evolution of $T_{m}$. However, the VMCM framework is generic and works just as seamlessly for problems involving curved crack propagation and mixed-mode evolution. ${ }^{8}$

${ }^{\text {e}}$ For this class of materials the simulations are more sensitive to the values of $T_{n_{0}}$ and $\mathscr{R}_{a v g}$, than the shape of the tractions separation curve.

${ }^{f}$ The small pre-peak stiffness difference in the experimental curves of some specimens is due to some amount of material crushing occurring at the loading points, and the post-peak variations are an artifact of the stochastic nature of failure in these materials.
} 
Having presented the relevance of VMCM and $\mathscr{R}_{\text {avg }}$, we now turn to the actual mechanics of the observed scaling behavior: Why does the fracture resistance (and hence $\mathscr{R}_{\text {avg }}$ ) increase with specimen size? As a start, we use VMCM simulations to study the effect of high fracture resistance on the $P \Delta$ response of this material model. Figure 13 shows the effect of varying $\mathscr{R}_{\text {avg }}$ on the peak $P \Delta$ response of the specimens. As seen, increase in $\mathscr{R}_{\text {avg }}$ leads to plateauing of the peak response. The peak load increases only slightly, but the tendency for softening past the peak is suppressed. One inference from this observation is that in this class of materials, high fracture resistance has only limited influence in increasing the peak load carrying ability of the specimens. Beyond a certain range of fracture resistance, the specimen geometry starts to play a significant role. This is evident in Figure 14, where the bridging zone formation and movement is depicted. As seen in the figure, after the formation of a FBZ, the zone propagates for some distance with almost a constant length. But as the FBZ approaches the end of the crack path, the compressive stresses encountered by the growing crack, caused due to bending of the SETB specimen about the loading point, inhibit further FBZ propagation, and hence the FBZ length decreases ${ }^{\mathrm{h}}$.

Based on the simulation results depicting the influence of $\mathscr{R}_{\text {avg }}$ on the $P \Delta$ response (Figure 11,13) and the FBZ formation (Figure 14), we present the following assessment of the failure behavior of this class of materials. It appears that this class of material is capable of achieving a very large bridging zone length, when their is no geometric constraint (both, ahead of the crack tip, and in the crack wake undergoing fiber pullout) on its natural bridging zone formation tendency. The corresponding maximum "full bridging zone" length is referred to as the "free bridging zone" (FrBZ) length, which may be considered a material property. In this FrBZ, a multitude of mechanisms (fiber failure, debounding, pullout) are active over large lengths, leading to a high fracture resistance. But in specimens with sizes comparable to these large lengths, the FrBZ formation mechanisms are inhibited and the specimen fails to manifest the FrBZ, but instead forms a smaller FBZ. Smaller the specimen size, larger the inhibition to free bridging zone formation, and thereby, smaller the corresponding FBZ length manifested. This reduced FBZ length leads to lower fracture resistance, and hence lower peak load.

\section{Discussion}

The material in consideration is composed of a quasi-brittle matrix and reinforcing carbon fibers. For this material, experiments show at first a matrix crack forming, leading to enhanced loading on the adjoining fibers, which suffer greater strain until they reach their failure stress. Ultimate failure occurs at weak points either at the matrix crack interface or inside the surrounding matrix. Fiber failure at the matrix crack interface leads to complete loss of load bearing capacity at the respective fiber. Hence this can be treated as the equivalent of a free crack surface in metals. But if the fiber fails at a weak point inside the intact matrix, it leads to fiber pull out, which involves adhesive bonding failure and frictional resistance to the fiber movement. ${ }^{1}$ As stated earlier, the process zone over which these mechanisms occur is termed the FBZ. These additional dissipation mechanisms contribute to an increase in fracture resistance until the fiber is completely pulled out of the matrix. These mechanisms are schematically depicted in Figure VI. Theoretical models have been developed to study fiber failure and pullout, and its effect on fracture resistance. ${ }^{1-4}$ These models are useful in gaining insight to the individual contributions of various constituent mechanisms towards fracture resistance, but in a real material the observed fracture resistance is a combination of these processes. The actual crack path formation is significantly stochastic, and it is prohibitively difficult, if not impossible, to delineate the contribution of each process. It is stressed here that in metals the energy dissipation is localized to the crack surfaces, which is the case also with matrix cracking. But the process of fiber failure and pullout leads to non-local dissipation spread out across significant portions of the specimen under consideration. Further complications arise because this globalized dissipation is dependent on loading

\footnotetext{
gEven though the linear traction separation law simplifies a set of complex failure mechanisms, the dissipated energy is well-represented. This quantity appears to control the fit with experimental $P \Delta$ curves.

${ }^{\mathrm{h}}$ The maximum FBZ length corresponds to the peak load. Any further progress of the FBZ has to be accompanied by a reduction in the FBZ length, and hence drop in $P$, to satisfy force and moment equilibrium of the specimen. Any further reduction in FBZ length due to compressive stresses ahead of the crack tip, is in addition to the reduction of the FBZ length necessitated due to equilibrium conditions.
} 
conditions and crack path orientation with respect to the fiber layup. The magnitude and non-locality of dissipation depends on the stress field. This lack of objectiveness runs contrary to the understanding of fracture in metals, where energy of surface creation is a material property and the sole contributor to the fracture resistance. Hence in metals fracture resistance is a material property referred to as the fracture toughness, but in this class of composites fracture resistance is an evolving, geometry, loading and crack orientation dependent quantity. It is pointed out here that though composites are classified as "materials" in a general context, and are modeled as appropriately homogenized continua, for failure evolution they need to be considered as "structures" rather than as materials. This consideration will bring in the appropriate length scales that dictate failure through mechanism-based micromechanical models. For instance, in compression failure of unidirectional composites, micromechanical models have successfully shown the initiation and evolution of fiber micro-buckling leading to kink banding failure. ${ }^{42}$ This lack of objectivity in fracture resistance at first seems to rule out a predictive capability for failure of these materials. However as shown in Section IV(B) average estimates of fracture resistance, $\mathscr{R}_{\text {avg }}$, significantly reproduce the observed failure response.

In Section IV(B), the effect of varying $\mathscr{R}_{\text {avg }}$ on the peak load of the specimen was briefly discussed. Figure 13 depicts the limited influence of $\mathscr{R}_{\text {avg }}$ in increasing the peak load of the specimen. As evident from the figure, beyond a certain range, increase in $\mathscr{R}_{\text {avg }}$ causes a plateauing of the $P \Delta$ response for the following reasons:

(1) Another parameter, $T_{n_{0}}$ (Equation 12, Figure 8), also influences the peak load magnitude. For any given FBZ length, the load carrying ability of the FBZ may be determined by adding the instantaneous load carried by all the individual fibers making up the FBZ. But the instantaneous load $\left(T_{n}\right)$ carried by each fiber is dictated by Equation 12, and thus $T_{n_{0}}$ influences the peak load of the specimen.

(2) Though $\mathscr{R}_{\text {avg }}$ and $T_{n_{0}}$ determine the maximum load carrying ability of the material, the actual evolution of the $P \Delta$ response has to be in accordance with the force, moment equilibrium conditions.

The concept of FrBZ has been introduced in the previous section. Here we expand on that discussion. As the specimen size increases, the potential crack path available for initial FBZ formation increases, so in larger specimens the material approaches its uninhibited $\mathscr{R}_{\text {avg }}$, and tends to realize its full FrBZ forming ability, hence higher peak loads. But at some characteristic geometric size, the material fully realizes its FrBZ, and at this stage manifests its maximum $\mathscr{R}_{\text {avg }}$ (referred to as $\mathscr{R}_{\max }$ ). For specimens sizes larger than this characteristic geometric size, the measured $\mathscr{R}_{\text {avg }}$ value equals $\mathscr{R}_{\max }$. $\mathscr{R}_{\max }$ depicts the "free" behavior of the composite structure for this particular crack evolution configuration (pure Mode-I), but it may be of little direct practical significance, as in most crack propagation problems the material will not have its "free" conditions, and the crack evolution may be different (mixed-mode). Other configurations will have different characteristic FrBZ and $\mathscr{R}_{\max }$. Hence it is important for designers to have a clear understanding of the FrBZ evolution and its dependence on specimen size and crack configuration. It is pointed out here that more extensive experimentation on larger size specimens of different geometries are required to substantiate this hypothesis on the behavior of this class of materials. Identification of a converged FrBZ and $\mathscr{R}_{\max }$ are the key to this understanding.

It is instructive to place the results that have been presented in the context of energetic size effect laws that have been developed by Bazant et al. ${ }^{23}$ When geometrically scaled specimens are stressed to failure, as described earlier, it has been shown that many types of quasi-brittle materials obey a type 2 size effect law that is described by Bazant et al. ${ }^{23}$ As shown there, performing a regression analysis of just the maximum loads for the SETB specimens (Figure 4) leads to estimates of FrBZ length and $\mathscr{R}_{\max }$ in accordance with the scaling law. Using this $\mathscr{R}_{\max }$ value as input, the VMCM simulations predict the FrBZ length which is in good agreement with the scaling law estimate $\left(\mathrm{FrBZ}_{V M C M} / \mathrm{FrBZ}_{\text {ScalingLaw }}=1.08\right)$. This comparison adds further confidence and validity to the findings presented here through the use of the VMCM method.

\section{Conclusion}

The results presented and the ensuing discussion demonstrate that:

(1) The material under consideration, unlike in metals, exhibits geometry, loading dependent fracture resistance. 
(2) Averaged fracture resistance $\left(\mathscr{R}_{\text {avg }}\right)$ values significantly reflect the macroscopic fracture resistance of these specimens.

(3) The existence of a "Free Bridging Zone" (FrBZ) and maximum fracture resistance $\left(\mathscr{R}_{\max }\right)$ are the characteristic of this material, and the geometric inhibition on FrBZ evolution and thereby $\mathscr{R}_{\max }$ manifestation is what leads to the observed scaling behavior. Not accounting for the existence of FrBZ and $\mathscr{R}_{\text {max }}$ posses the grave risk of incorrect predictions of strength and reliability; because in some class of materials, like the one being considered here, the FBZ formation may wrongly signal the formation of a converged process zone (FrBZ).

\section{Acknowledgments}

This work has benefited from the financial support of Collier Research Corporation, through a NASA NRA award. The interest and encouragement of Steven M. Arnold and Brett Bednarcyk of NASA Glenn Research Center is gratefully acknowledged.

\section{References}

${ }^{1}$ Cooper GA, "The fracture toughness of composites reinforced with weakened fibers", Journal of Materials Science, $\mathbf{5}$, 645-654, 1970.

${ }^{2}$ Aveston J, Cooper GA \& Kelly A "The Properties of Fibre Composites", Conference Proceedings, National Physical Laboratory (IPC Science and Technology Press Ltd), 1971.

${ }^{3}$ Aveston J \& Kelly A, "Theory of multiple fracture of fibrous composites", Journal of Materials Science, 8, 352-362, 1973.

${ }^{4}$ Cox BN, "Extrinsic factors in the mechanics of bridged cracks", Acta metallurgica et materialia, 39, 1189-1201, 1991.

${ }^{5}$ Simo JC \& Rifai MS, "A Class of mixed assumed strain methods and the method of incompatible modes", International Journal for Numerical Methods in Engineering, 29, 1595-1638, 1990.

${ }^{6}$ Simo JC \& Armero F, "Geometrically non-linear enhanced strain mixed methods and the method of incompatible modes", International Journal for Numerical Methods in Engineering, 33, 413-1449, 1992.

${ }^{7}$ Garikipati K, "On Strong Discontinuities in Inelastic Solids and Their Numerical Simulation", Ph.D. Thesis, Stanford University, Stanford, California, USA, 1996.

${ }^{8}$ Garikipati K, "A variational multiscale method to embed micromechanical surface laws in the macromechanical continuum formulation", Computational Modeling in Engineering and Sciences, 3, 175-184, 2002.

${ }^{9}$ F. Armero \& K. Garikipati, "An analysis of strong discontinuities in multiplicative finite strain plasticity and their relation with the numerical simulation of strain localization in solids", International Journal of Solids and Structures, 33, 28632885, 1996.

${ }^{10}$ T.Belytschko, N. Moes, S. Usui \& C. Parimi, "Arbitrary discontinuities in finite elements", International Journal for Numerical Methods in Engineering, 50, 9931013, 2001.

${ }^{11}$ T.Belytschko, H. Chen, J.X. Xu \& K. Garikipati, "Dynamic crack propagation based on loss of hyperbolicity and a new discontinuous enrichment", International Journal for Numerical Methods in Engineering, 58, 18731905, 2003.

${ }^{12} \mathrm{~K}$. Garikipati \& T.J.R. Hughes " A study of strain-localization in a multiple scale framework. The one dimensional problem", Computer Methods in Applied Mechanics and Engineering, 159, 193222, 1998.

${ }^{13}$ T.C. Gasser \& G.A. Holzapfel, "Geometrically non-linear and consistently linearized embedded strong discontinuity models for $3 D$ problems with an application to the dissection analysis of soft biological tissues", Computer Methods in Applied Mechanics and Engineering, 192, 50595098, 2003.

${ }^{14} \mathrm{M}$. Jirasek, "Comparative study on finite elements with embedded discontinuities", Computer Methods in Applied Mechanics and Engineering, 188, 307330", 2000.

${ }^{15} \mathrm{~J}$. Mosler \& G. Meschke, "Embedded crack vs. smeared crack models: a comparison of elementwise discontinuous crack path approaches with emphasis on mesh bias", Computer Methods in Applied Mechanics and Engineering, 193, 33513375, 2004.

${ }^{16}$ J. Oliver \& A.E. Huespe, "Continuum approach to material failure in strong discontinuity settings", Computer Methods in Applied Mechanics and Engineering, 193, 31953220, 2004.

${ }^{17}$ R.L. Borja \& R.A. Regueiro, "A finite element model for strain localization analysis of strongly discontinuous fields based on standard Galerkin approximation", Computer Methods in Applied Mechanics and Engineering, 190, 15291549, 2000.

${ }^{18}$ N. Moes, N. Sukumar, B. Moran \& T. Belytschko, "An extended finite element method (X-FEM) for two and threedimensional crack modelling", Presented at ECCOMAS 2000, Barcelona, Spain, 2000.

${ }^{19}$ G.N. Wells \& L.J. Sluys, "A new method for modelling cohesive cracks using finite elements", nternational Journal for Numerical Methods in Engineering, 50, 26672682, 2001.

${ }^{20}$ O. Nguyen, E.A. Repetto, M. Ortiz \& R.A. Radovitzky, "A cohesive model of fatigue crack growth", International Journal of Fracture, 110, 351-369, 2001.

${ }^{21}$ Oliver J, Huespe AE \& Sanchez PJ, "A comparative study on finite elements for capturing strong discontinuities : E-FEM vs X-FEM", Computer methods in applied mechanics and engineering, 195, 4732-4752, 2006.

${ }^{22}$ Siva S. Rudraraju, Ryan Vignes, Amit Salvi, Garikipati K \& Anthony M. Waas, "A multiscale crack path predicting computational method for laminated fiber reinforced composites", Proceedings of the 49th AIAA/ASME/ASCE/AHS/ASC Structures, Structural Dynamics, and Materials Conference, Schaumburg, Illinois, USA, 2008.

\section{9 of 18}

American Institute of Aeronautics and Astronautics 
${ }^{23}$ Zdenek P. Baant \& Mohammad T. Kazemi, "Size Effect in Fracture of Ceramics and Its Use To Determine Fracture Energy and Effective Process Zone Length", Journal of the American Ceramic Society", 73, 1841-1853, 1990.

${ }^{24}$ Scheider I \& Brocks W, "The effect of the traction-separation law on the results of cohesive zone crack propagation analyses", Fracture and Damage Mechanics, Proceedings of the 3rd International Conference on Fracture and Damage Mechanics, Trans Tech Publications, Zurich, Switzerland, 2003.

${ }^{25}$ Barenblatt GI, "The mathematical theory of equilibrium cracks in brittle fracture", Advances in Applied Mechanics, 7, 55129, 1962.

${ }^{26}$ Dugdale DS, "Yielding of steel sheets containing slits", Journal of the Mechanics and Physics of Solids, 8, $100108,1960$.

${ }^{27}$ Hillerborg A, Modeer M \& Petersson PE, "Analysis of crack formation and crack growth in concrete by means of fracture mechanics and finite elements", Cement and Concrete Research, 6, 773782, 1976.

${ }^{28}$ Pietruszczak S \& Mroz Z, "Finite element analysis of deformation of strain softening materials", International Journal for Numerical Methods in Engineering, 17, 327-334, 1981.

${ }^{29}$ Ungsuwarungsri T \& Knauss WG, "The Role of Damage-Softened Material Behavior in the fracture of composites and adhesives", International Journal of Fracture, 35, 221-241, 1987.

${ }^{30}$ Tvergaard V \& Hutchinson JW, "The relation between crack growth resistance and fracture parameters in elastic-plastic solids", Journal of the Mechanics and Physics of Solids, 40, 1377-1397, 1992.

${ }^{31}$ TSchellekens JCJ \& De Borst R, "On the numerical integration of interface elements", International Journal for Numerical Methods in Engineering, 36, 43-66, 1993.

${ }^{32} \mathrm{Xu}$ XP \& Needleman, "A. Numerical simulation of fast crack growth in brittle solids", Journal of the Mechanics and Physics of Solids, 42, 1397-1434, 1994.

${ }^{33}$ Camacho GT \& Ortiz M, "Computational modeling of impact damage in brittle materials", International Journal of Solids and Structures, 33, 2899-2938, 1996.

${ }^{34}$ Richard W. Hertzberg, "Deformation and fracture mechanics of engineering materials", Wiley, 1983.

${ }^{35}$ Xie D \& Biggers Jr. SB, "Strain energy release rate calculation for a moving delamination front of arbitrary shape based on virtual crack closure technique, Part I: Formulation and Validation", Engineering Fracture Mechanics, 73, 771-785, 2006.

${ }^{36}$ Xie D \& Biggers Jr. SB, "Strain energy release rate calculation for a moving delamination front of arbitrary shape based on virtual crack closure technique, Part II: Sensitivity Study on Modeling Details", Engineering Fracture Mechanics, $\mathbf{7 3}$, 786-801, 2006.

${ }^{37}$ Xie D, Anthony M. Waas, Shahwan KW, Schroeder JA \& Boeman RG, "Computation of energy release rates for kinking cracks based on virtual crack closure technique", ECMES-Computer Modeling in Engineering and Sciences, 2004.

${ }^{38}$ Xie D, Chung J, Anthony M. Waas, Shahwan KW, Schroeder JA, Boeman RG, Kunc V, Klett LB, "Failure analysis of adhesively bonded structures: from coupon level data to structure level predictions and verification", International Journal of Fracture, 134, 231-250, 2005.

${ }^{39}$ Xie D \& Anthony M. Waas, "Discrete cohesive zone model for mixed-mode fracture using finite element analysis", Engineering Fracture Mechanics, 73, 1783-1796, 2006.

${ }^{40}$ Amit Salvi, Anthony M. Waas \& Caliskan A, "Energy absorption and damage propagation in $2 D$ triaxially braided carbon fiber composites: Effects of in situ matrix properties", Journal of Materials Science, 43, 5168-5184, 2008.

${ }^{41}$ Xie D, Amit Salvi, Ce Sun, Anthony M. Waas, \& Caliskan A, "Discrete cohesive zone model to simulate static fracture in 2-D triaxially braided carbon fiber composites", Journal Composite Materials, 40, 1-22, 2006.

${ }^{42}$ Shiladitya Basu, Anthony M. Waas \& Damodar R. Ambur, "A macroscopic model for kink banding instabilities in fiber composites", Journal of Mechanics of Materials and Structures, 1, 2006.

${ }^{43}$ T. Andersson \& U. Stigh, "The stresselongation relation for an adhesive layer loaded in peel using equilibrium of energetic forces", International Journal of Solids and Structures, 41, 413-434, 2004.

${ }^{44} \mathrm{BF}$ Sorensen \& TK Jacobsen, "Large-scale bridging in composites: R-curves and bridging laws", Composites Part A Applied Science and Manufacturing, 29, 1443-1451, 1998.

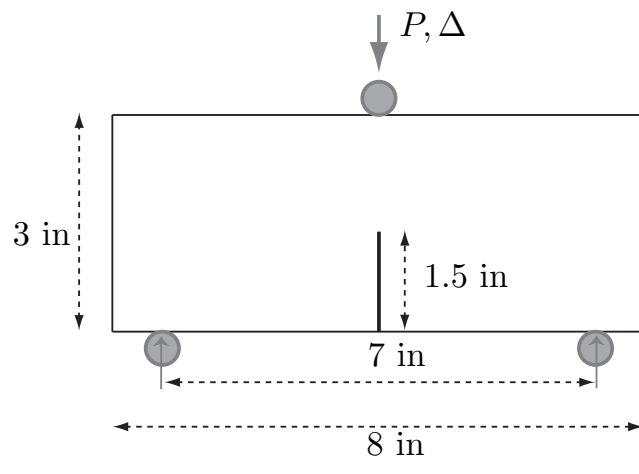

Size 1: X (as shown)

Size 2: $1.5 \mathrm{X}$

Size 3: $2 \mathrm{X}$

Size 4: $3 X$

Size 5: $4 X$

Figure 1. Single Edge Notch Bending(SETB) specimen configuration used for validating VMCM simulation results. Size 1 has the dimensions shown in figure, other sizes are scaled versions of this base size. All specimens have a thickness of $6.35 \mathrm{~mm}$. 

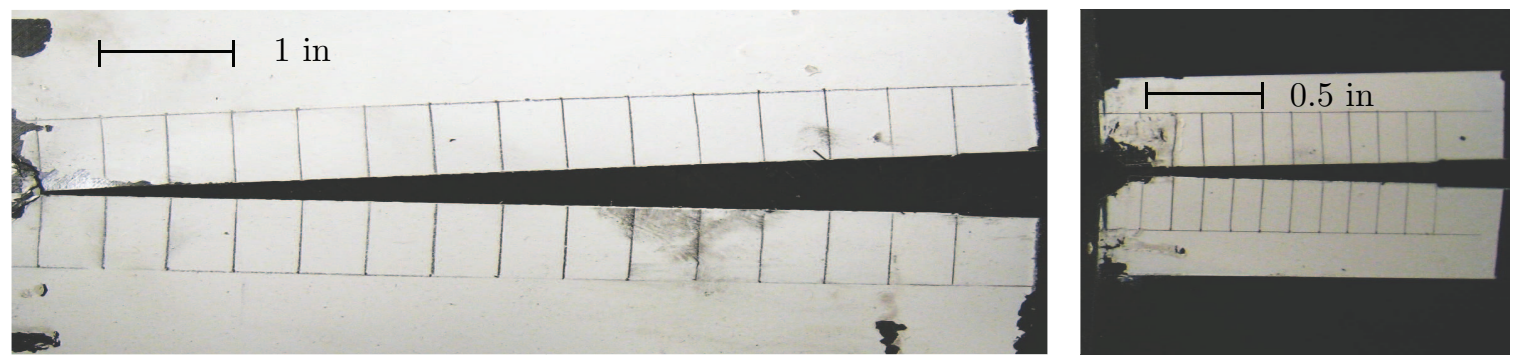

Crack opening in Size 5 specimen

Crack opening in Size 1 specimen

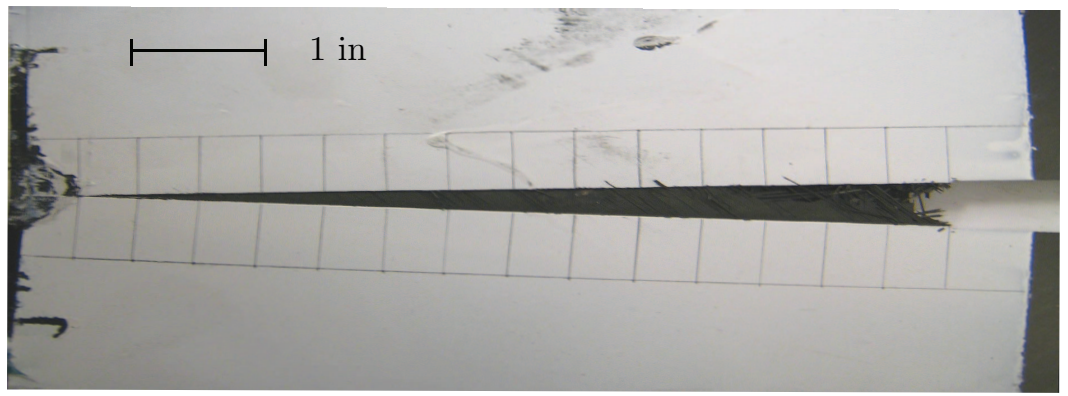

Fiber bridging in Size 5 specimen

Figure 2. Comparison of crack tip opening in failed SETB size-1 and size-5 specimens.

Table 1. Scaling observed in the SETB specimen experiments.

\begin{tabular}{|c|c|cc|c|}
\hline Size & $\begin{array}{c}\text { Geometry scaling } \\
\text { (Figure 1) }\end{array}$ & $\begin{array}{c}\text { Peak load } \\
P / P^{*}\end{array}$ & $\begin{array}{c}\text { Load point displacement } \\
\Delta / \Delta^{*}\end{array}$ & $\begin{array}{c}\text { Fracture resistance } \\
\mathscr{R}_{\text {avg }} / \mathscr{R}_{\text {avg }}^{*}\end{array}$ \\
\hline \hline 1 & 1 & 0.27 & 0.1 & 1.08 \\
2 & 1.5 & 0.4 & 0.15 & 1.23 \\
3 & 2 & 0.6 & 0.2 & 1.84 \\
4 & 3 & 0.81 & 0.28 & 2.46 \\
5 & 4 & 1.0 & 0.37 & 2.58 \\
\hline
\end{tabular}

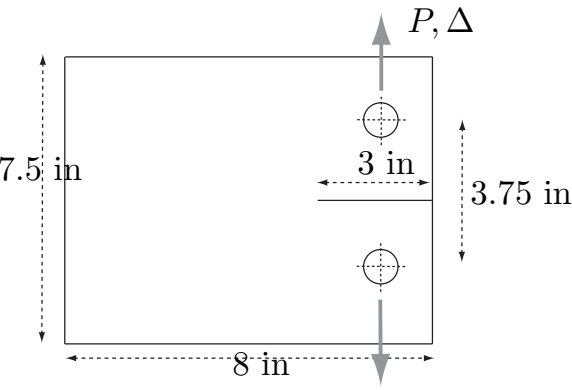

(a)

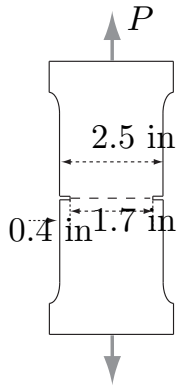

(b)

Figure 3. (a) Compact tension specimen (CTS) configuration used to obtain $\mathscr{R}_{a v g}$ for Mode I crack propagation. (b) Double notch tension specimen configuration used to obtain critical cohesive stress value. 


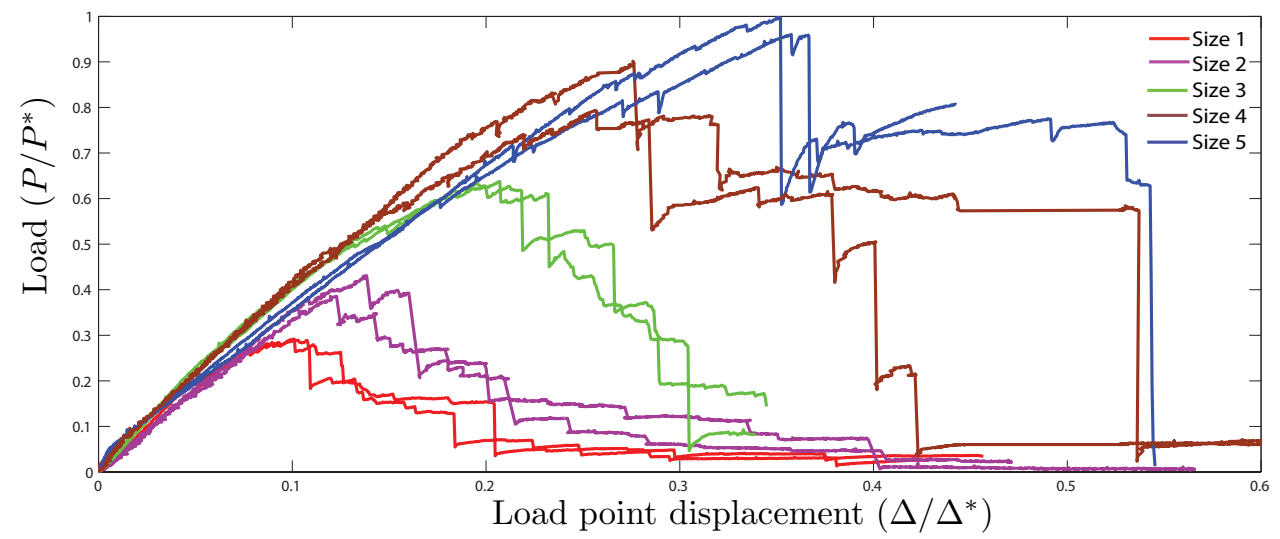

Figure 4. Experimental Load $(P)$ - Load point displacement $(\Delta)$ curves obtained for various sizes of SETB specimens. Multiple specimens of each size were tested to capture the envelope of the failure response. $P^{*}$ and $\Delta^{*}$ are fixed reference values.

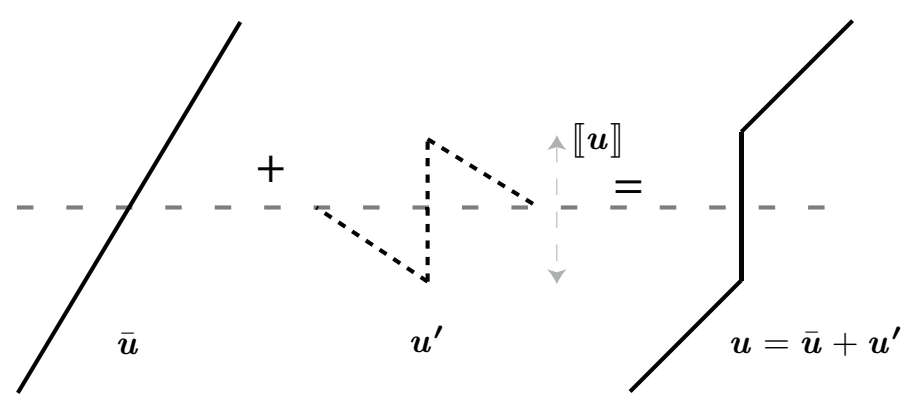

Figure 5. Schematic of scale separation. $\bar{u}$ is the coarse scale displacement field and $u^{\prime}$ is the local fine scale enhancement.

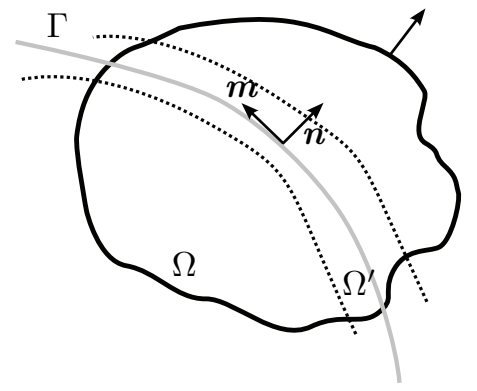

Figure 6. Decomposition of continuum body into region where coarse scale and fine scale displacements are defined. $\Omega$ is the domain of the problem, $\Gamma$ is the displacement discontinuity (crack), $\Omega^{\prime}$ is the support for the displacement discontinuity and $n, m$ are the normal and tangent to $\Gamma$ respectively. 


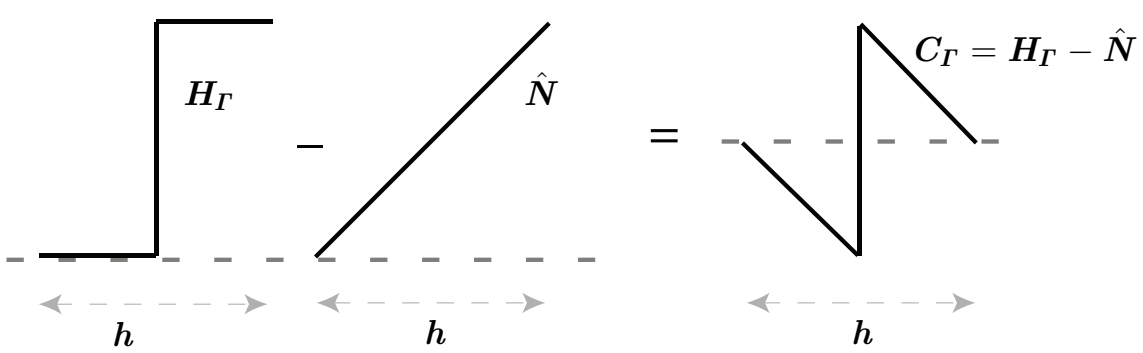

Figure 7. Discontinuous shape function used to resolve the displacement jump shown in a one-dimensional setting. It is constructed by superimposing a discontinuous function, $H_{\Gamma}$, on a regular polynomial, $\hat{N}$. $h$ is the element dimension.

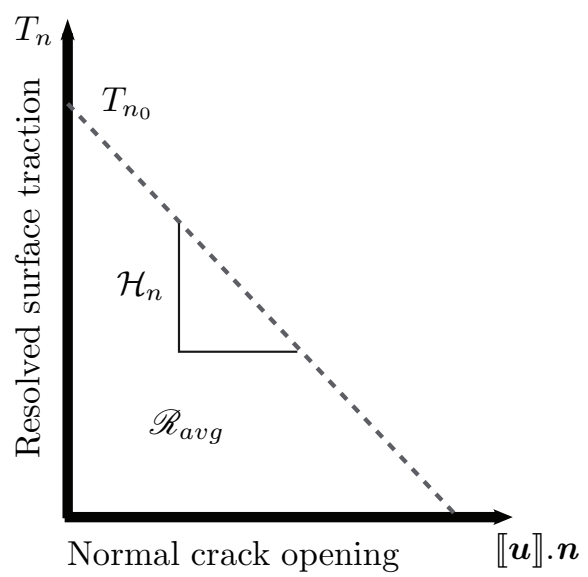

Figure 8. Linear micro-mechanical surface law for normal crack opening

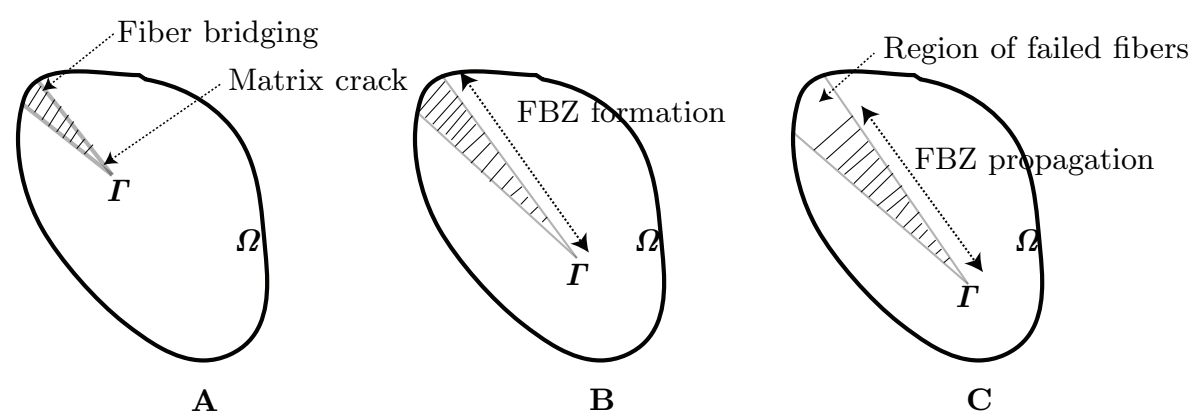

Figure 9. Stages involved in fiber composite cracking. A: Matrix cracking - Fiber bridging, B: FBZ formation, C: FBZ propagation. 


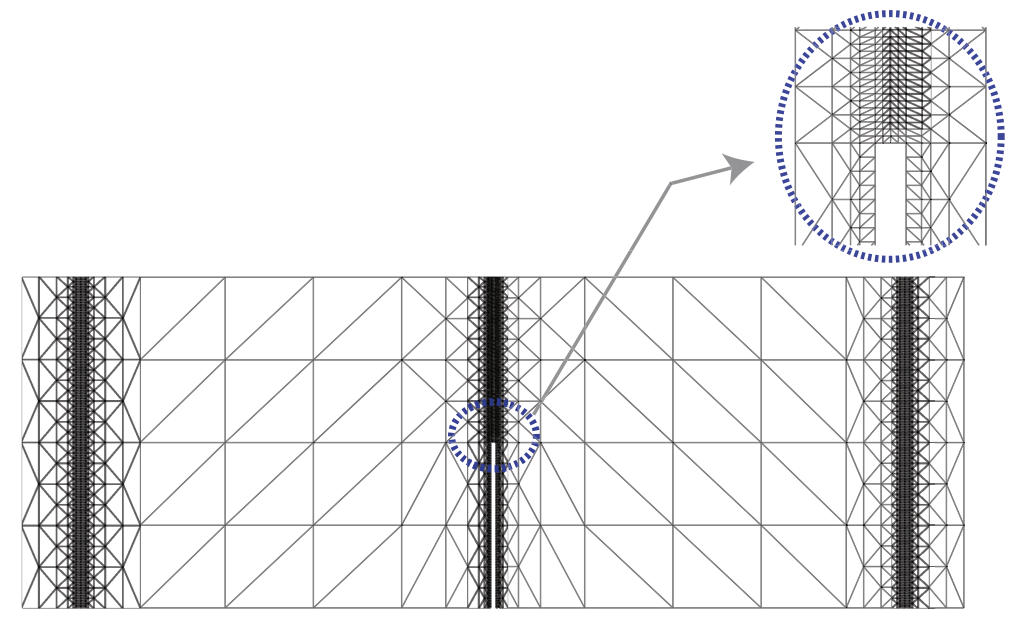

(a)

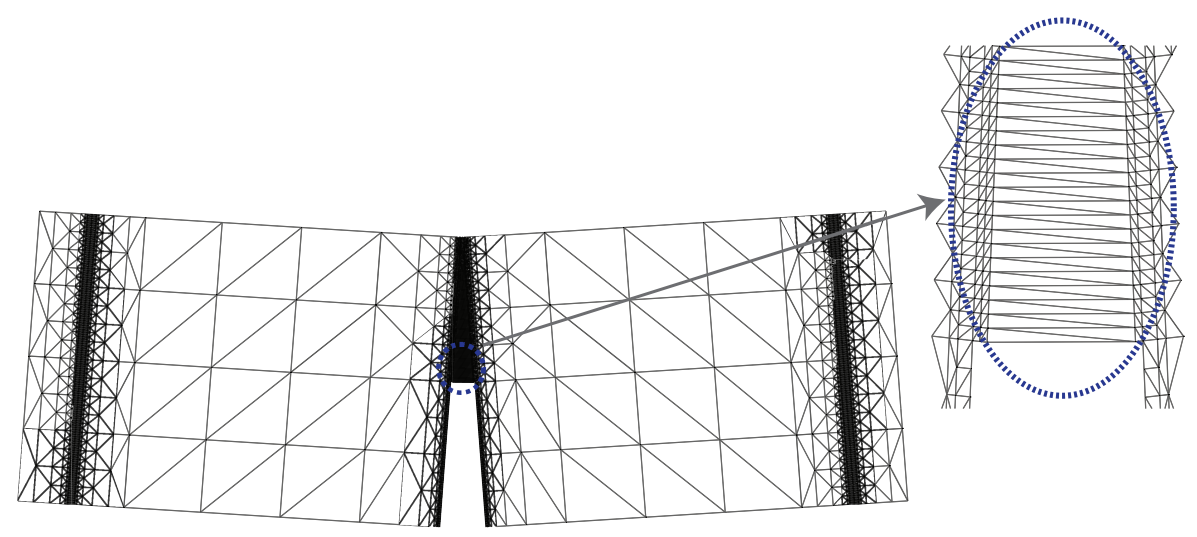

(b)

Figure 10. SETB specimen mesh, with crack tip elements magnified in inset. (a) Original mesh (b) Deformed mesh 
Size 1

A

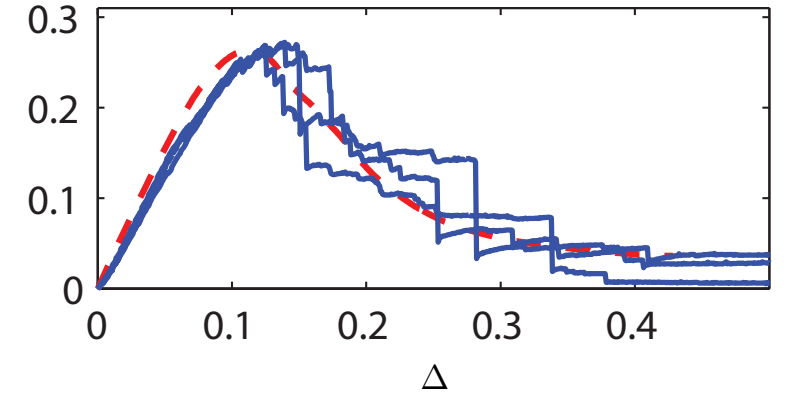

Size 3

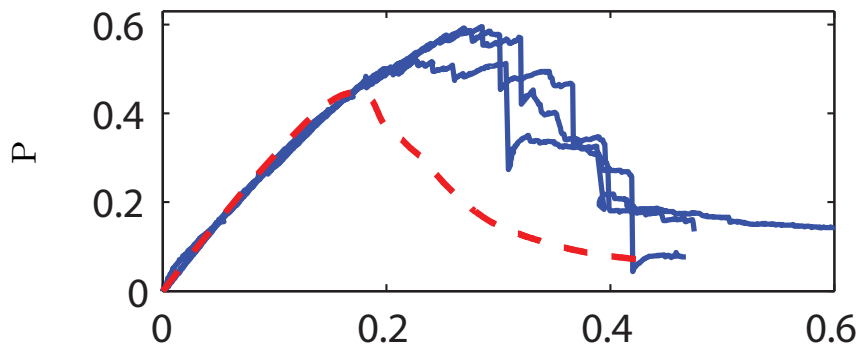

$\Delta$

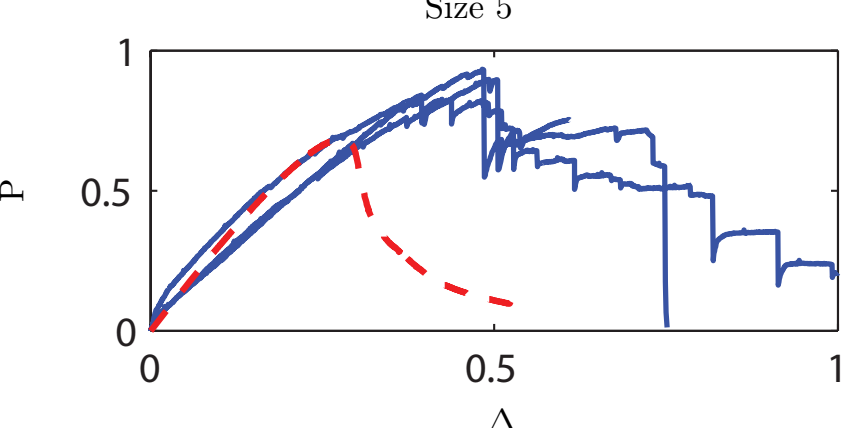

A.

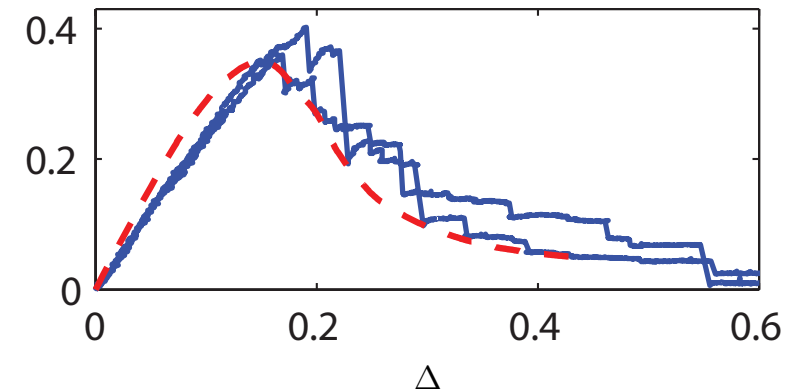

Size 4

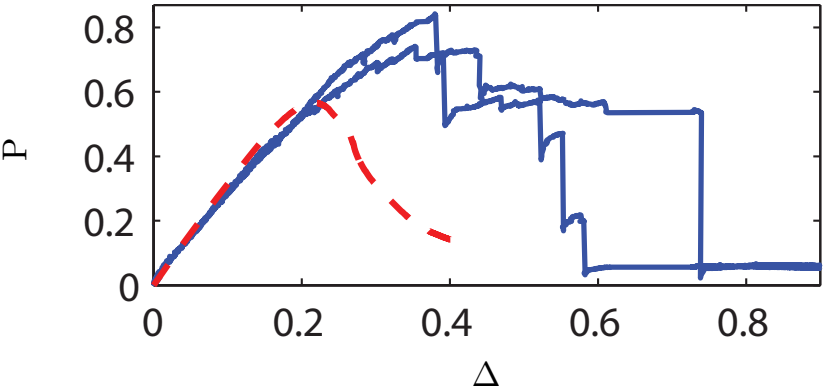

- - - : VMCM Experiments

Figure 11. Load-Displacement $(P \Delta)$ response, extracted from VMCM simulations with a constant, $\mathscr{R}_{\text {avg }}=\mathscr{R}_{\text {avg }}^{*}$, input for Size 1-5 SETB specimens, compared with the experimental curves. 
Size 1

A

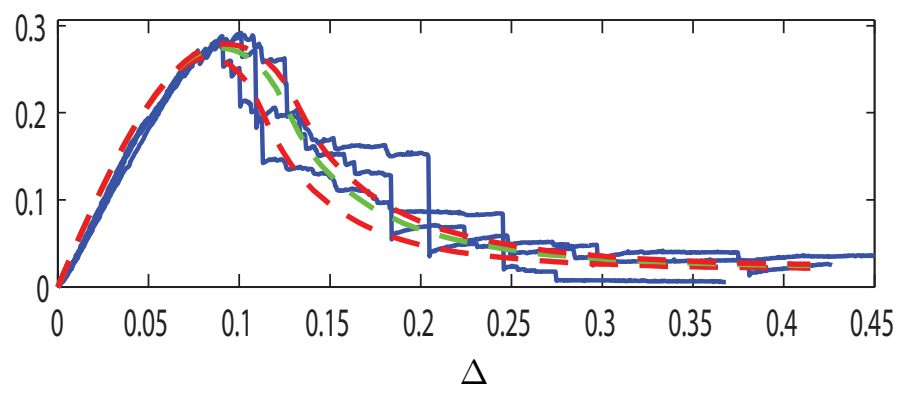

Size 3

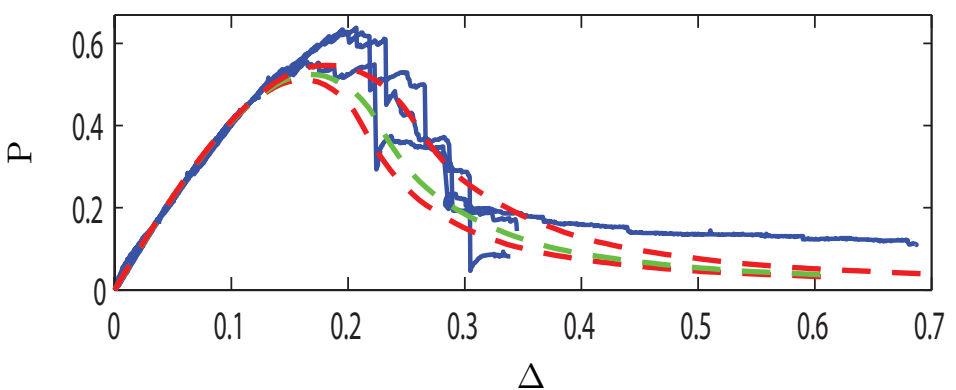

Size 2

a

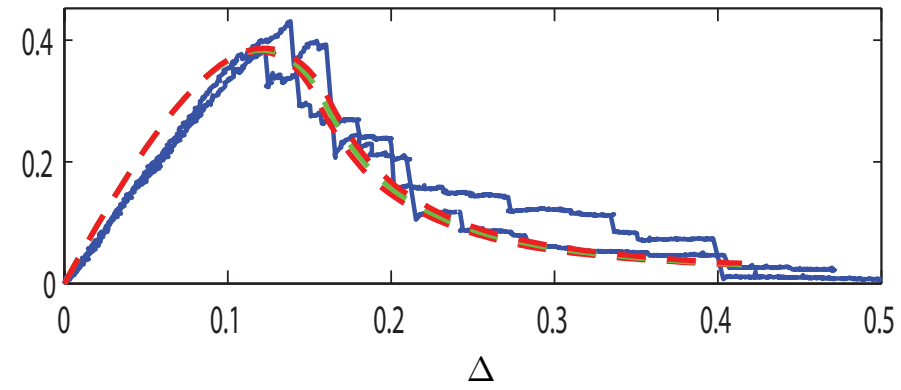

Size 4

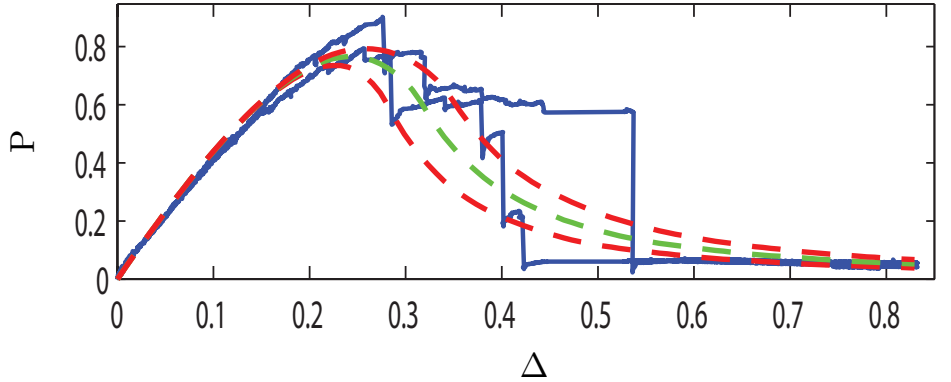

Size 5

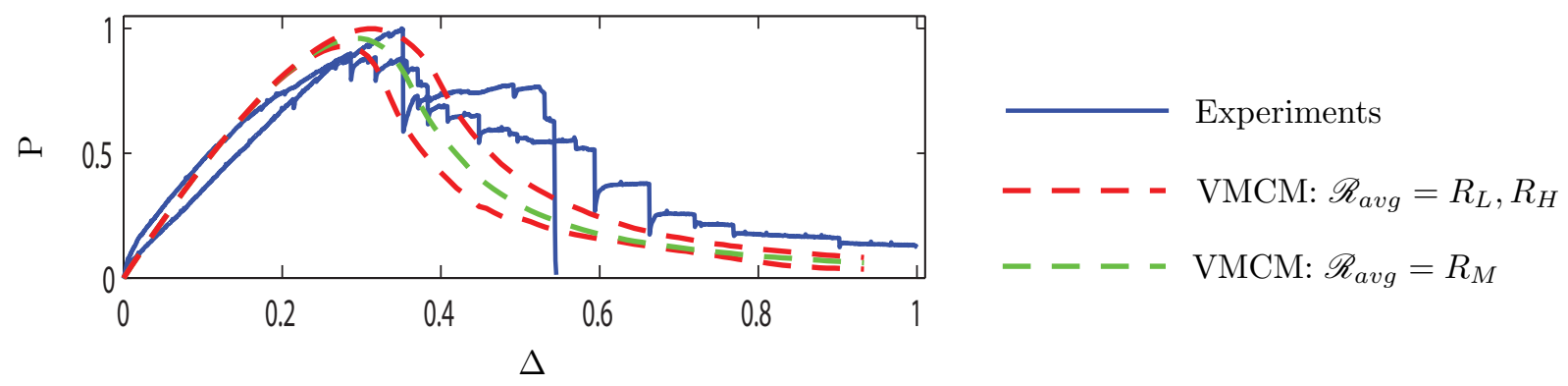

Figure 12. Load-Displacement $(P \Delta)$ response obtained from VMCM simulations of Size 1-5 SETB specimens with experimentally determined $\mathscr{R}_{a v g}$ values (Table 1), compared to their respective experimental curves. For a particular specimen size, $R_{L}$ and $R_{H}$ are the least and highest values of fracture resistance obtained from the multiple experimental $P \Delta$ curves, $R_{M}$ is the average of the fracture resistance of each of the multiple experimental $P \Delta$ curves. $R_{L}$ corresponds to the curve exhibiting least toughness and $R_{H}$ corresponds to the curve exhibiting the highest toughness. 
Size 1

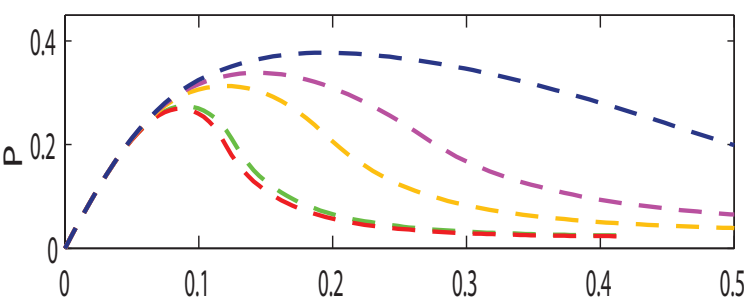

$\Delta$

Size 3

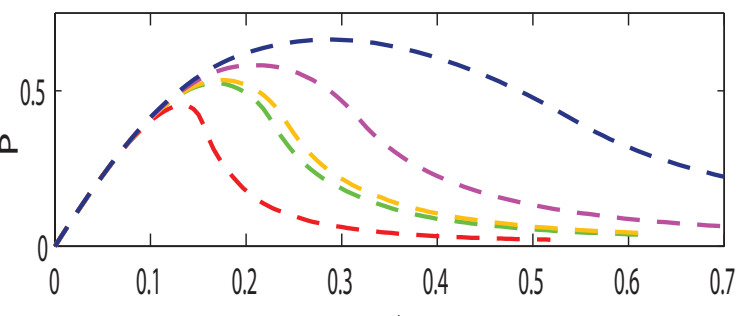

$\Delta$

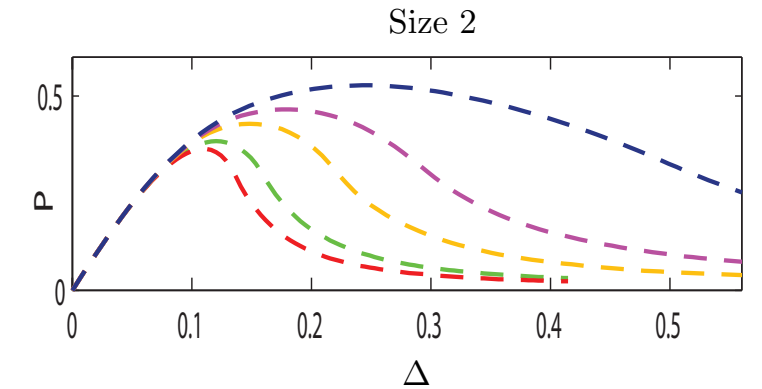

Size 4

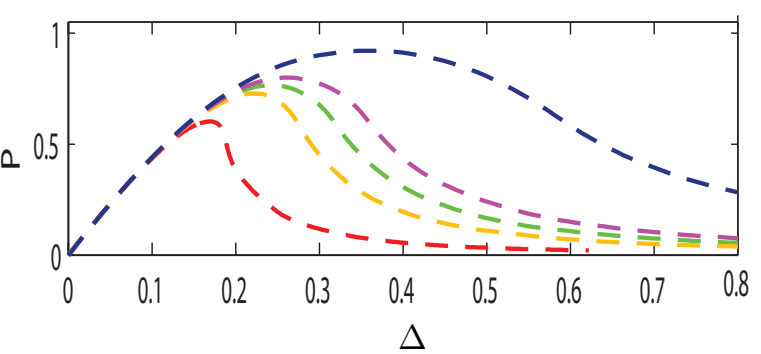

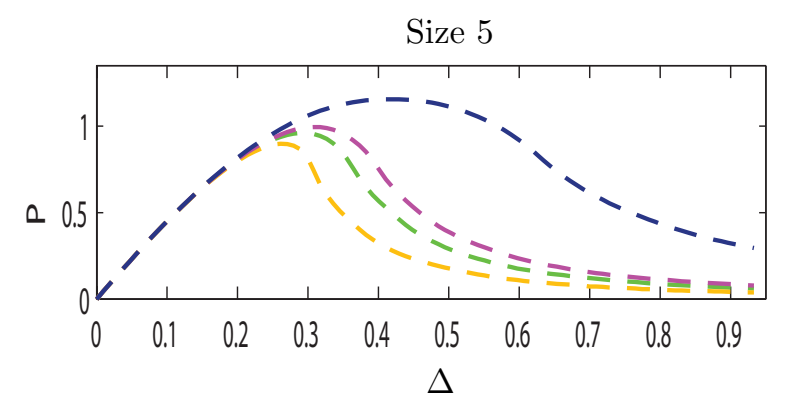

$----\mathscr{R}_{\text {avg }}$ : Experimental

$----\mathscr{R}_{a v g}=\mathscr{R}_{a v g}^{*}$

$----\mathscr{R}_{\text {avg }}=2 \mathscr{R}_{\text {avg }}^{*}$

$----\mathscr{R}_{\text {avg }}=3 \mathscr{R}_{\text {avg }}^{*}$

$---\mathscr{R}_{\text {avg }}=6 \mathscr{R}_{\text {avg }}^{*}$

Figure 13. Effect of increasing fracture resistance input on the Load-Displacement $(P \Delta)$ response of VMCM simulations. $\mathscr{R}_{a v g}^{*}$ is a constant reference value. Also plotted is the corresponding $P \Delta$ response obtained with the experimentally determined fracture resistance value. 

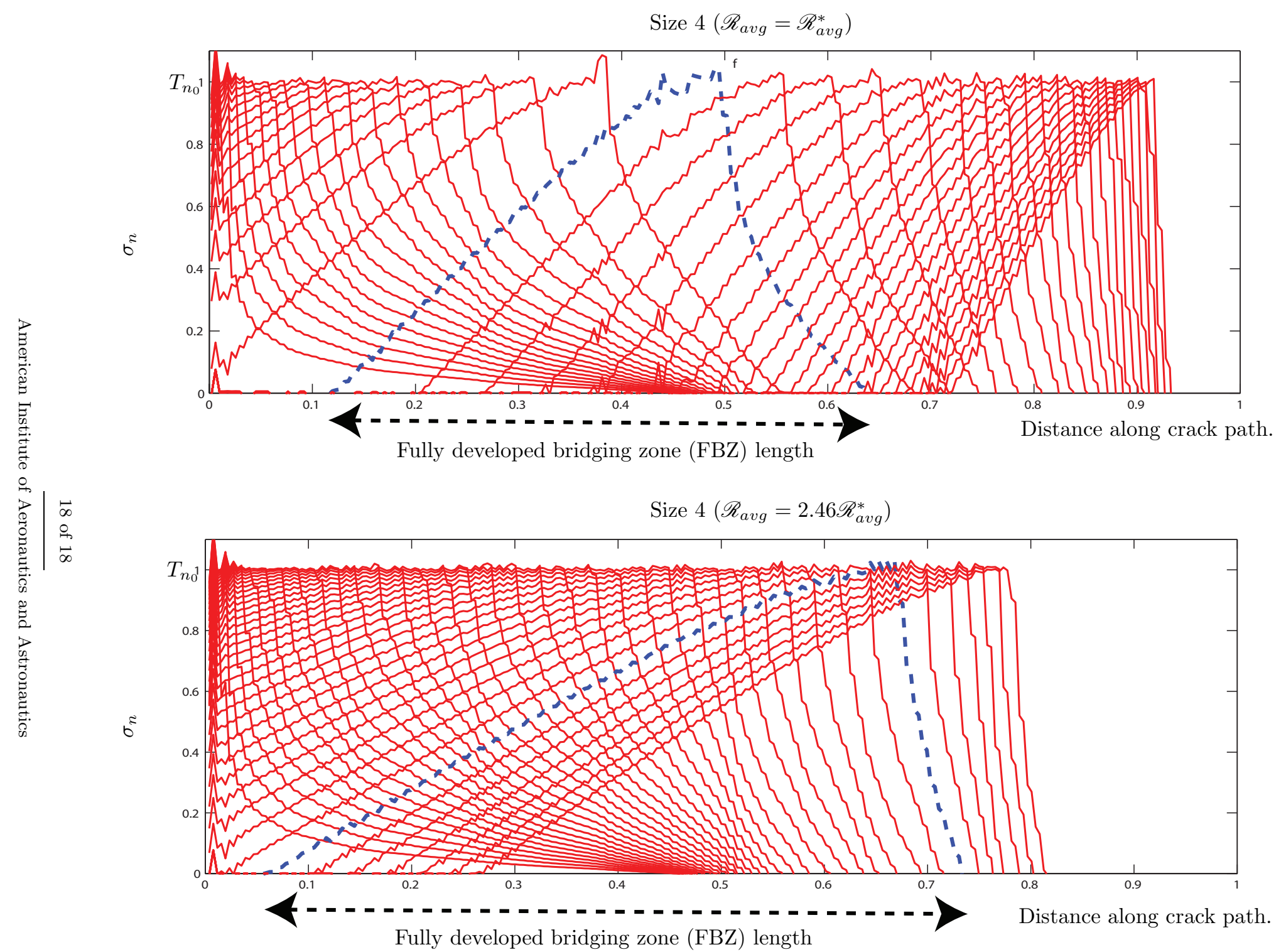

Figure 14. Snapshots of the normal stress $\sigma_{n}$ distribution along the crack path at different points in the loading cycle for Size-4 SETB specimen. The FBZ length labeled corresponds to the peak load in the loading cycle. Only the positive ordinate axes is shown. These figures give an estimate of the bridging zone length, and help in understanding bridging zone formation, stabilization and movement. 